\title{
Cryptosporidium Infection: Epidemiology, Pathogenesis, and Differential Diagnosis
}

\author{
Elisabetta Gerace ${ }^{1}$, Vincenzo Di Marco Lo Presti ${ }^{1}$ and Carmelo Biondo ${ }^{2 *}$ \\ ${ }^{1}$ Istituto Zooprofilattico Sperimentale della Sicilia A. Mirri, Italy \\ ${ }^{2}$ Laboratory of Microbiology and Parasitology, Departments of Human Pathology, University of Messina, Messina, Italy
}

Received: 08 Aug 2019; accepted: 16 Aug 2019

\begin{abstract}
Cryptosporidium is a protozoan that infects a wide variety of vertebrates, including humans, causing acute gastroenteritis. The disease manifests with abdominal pain and diarrhea similar to that of choleric infection. In the immunocompromised hosts, the parasite causes prolonged infections that can also be fatal. For this reason, cryptosporidiosis is considered one of riskiest opportunistic infections for patients with acquired immunodeficiency syndrome. The best way to control the infection in these patients is setting up sensitive and specific diagnostic tests for epidemiological surveillance and morbidity reduction. Here, we summarized the general aspects of Cryptosporidium infection focusing on available diagnostic tools used for the diagnosis of cryptosporidiosis. Molecular methods currently available for its detection and progress in the development of new diagnostics for cryptosporidiosis are also discussed.
\end{abstract}

Keywords: protozoan parasite, severe diarrhea, immunocompromised patients, differential diagnosis, molecular techniques

\section{Introduction}

Cryptosporidiosis is a worldwide infection caused by the protozoan Cryptosporidium, a parasite that infects many species of vertebrates, including humans, causing acute gastroenteritis, abdominal pain, and diarrhea [1]. Cryptosporidiosis is transmitted primarily through the fecal-oral route, i.e., by ingesting viable oocysts of animal and/or human origin, emitted with feces that contaminated food or water [2, 3]. Although the waterborne transmission of infectious pathogen is well documented, neither the natural reservoir nor the exact infection route of cryptosporidia is well-known [4].

Cryptosporidium was first discovered by Tyzzer in 1907 [5], but for a longtime, it was thought to be a non-pathogenic parasite. Only since 1976, it was recognized as an opportunistic pathogenic parasite, when 2 human cases of cryptosporidiosis were reported to be associated with diarrhea $[6,7]$. However, Cryptosporidium remained unknown as a significant human pathogen of acute diarrhea disease until 1982, when it was recognized as a causative agent of self-limiting diarrhea for the general population and a life-threatening disease for immunocompromised persons such as those receiving immunosuppressive agents and acquired immunodeficiency syndrome (AIDS) patients $[4,8,9]$.

The parasite completes its life cycle within a single host (monoxen cycle), alternating asexual and sexual reproduction $[3,5]$. Although more than 30 species have been included in the genus Cryptosporidium, only 2 species, namely, Cryptosporidium parvum and Cryptosporidium hominis, commonly infect humans [10-12]. C. parvum is responsible for most cattle infections, and consequently, it is considered to be responsible for most zoonotic infections in humans [10]. Cryptosporidium spp. exhibit little host specificity, and different members of this genus have been reported to infect

*Author for correspondence: Policlinico Universitario, $2^{\circ} \mathrm{p}$ Torre Biologica, 98125, Gazzi, Messina; E-mail: cbiondo@unime.it; Tel: +390902213322. multiple hosts, such as mammals, marsupials, birds, reptiles, and fish [13, 14]. The intracellular protozoan parasite Cryptosporidium is a human and veterinary pathogen - a member of the phylum Apicomplexa that include other pathogens such as Plasmodium spp., Eimeria spp., Neospora, Babesia, and Theileria [15]. However, in contrast to other parasitic protozoa of this phylum, such as Toxoplasma gondii or Plasmodium falciparum, the species of the genus Cryptosporidium cannot be cultivated in vitro [16]. Since there is no vaccine commercially available to prevent Cryptosporidium infection, and these parasites have certain characteristics that make them highly contagious (i.e., survival in the environment for a long time and resistance to chlorine-based disinfectants), the only way to avoid the spreading of the parasite to other people is the introduction of preventive measures to control the transmission of the germs that are shed in feces [12]. These precautions are especially important for people with weakened immune systems [17].

\section{Life Cycle and Epidemiology}

Infection is initiated by ingestion of oocysts, each of which contains 4 sporozoites that hatch at the intestinal level, releasing infectious sporozoites $[3,5]$. After excystation, these sporozoites are ingested into a modified host membrane separated from the cytoplasm by a dense layer; then, the location of parasites within the host is not intracellular but extracytoplasmic [18]. Within the parasitophorous vacuole, the parasite undergoes asexual or schizogony reproduction, leading to the production of 8 merozoites within a type I meront [19]. The merozoites can invade the neighboring epithelial cells and propagate the infection to other sites of the intestines. During this stage, the merozoites can undergo 2 distinct replicative cycles: an asexual stage characterized by multiplication of merozoites (type I meront) and production of thin-walled oocysts that autoinfect the host and/or a sexual stage with formation of type II meront, which, after differentiation in

This is an open-access article distributed under the terms of the Creative Commons Attribution-NonCommercial 4.0 International License (https://creativecommons.org/licenses/by-nc/4.0/), which permits unrestricted use, distribution, and reproduction in any medium for non-commercial purposes, provided the original author and source are credited, a link to the CC License is provided, and changes - if any - are indicated. 


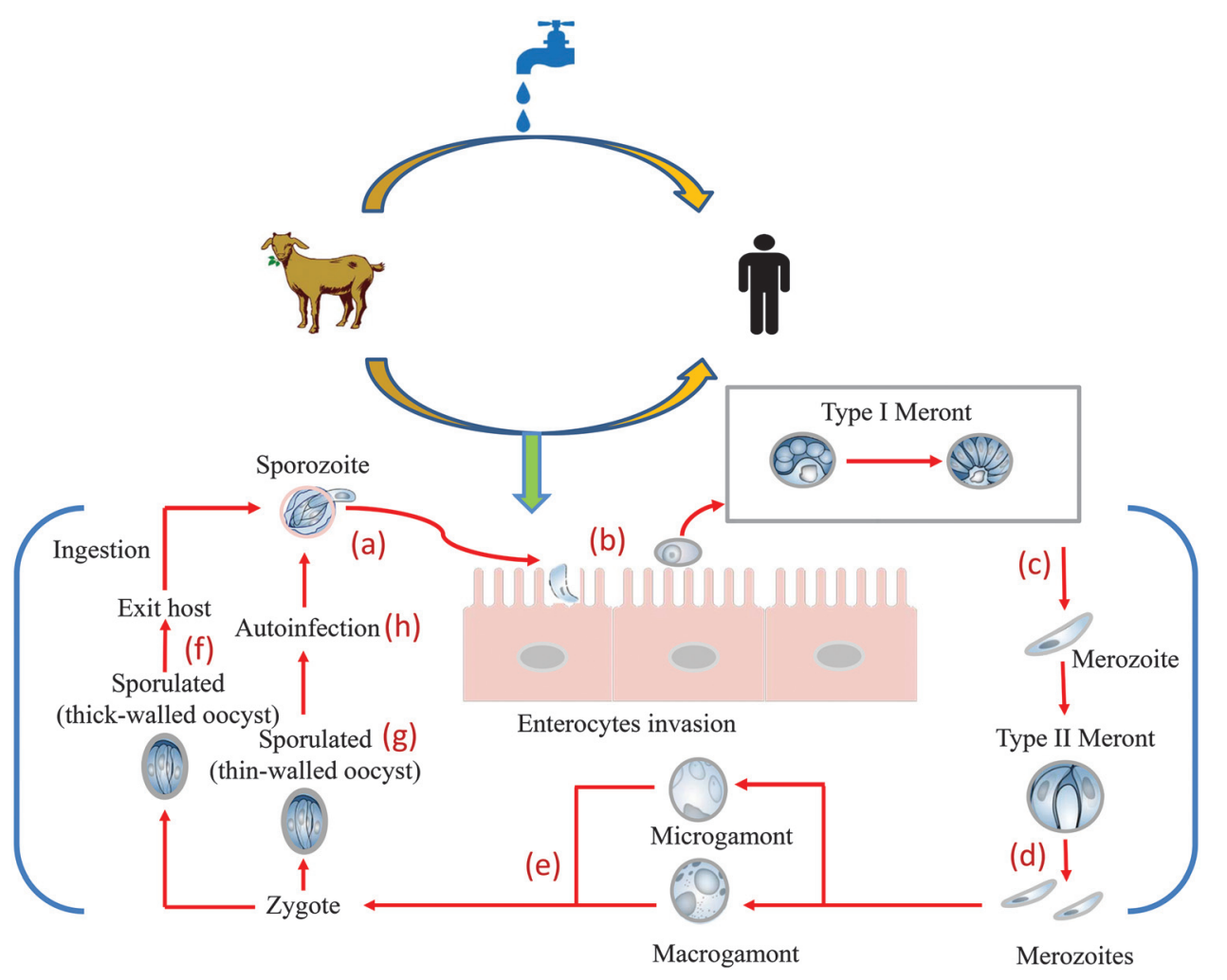

Figure 1. Cryptosporidium: source of infection and mode of transmission. Infection begins when an individual ingests protozoan oocysts that have been released into the feces from an infected human or animal. In the gastrointestinal tract, the oocysts are broken releasing 4 sporozoites (a) that invade the mucosa to establish a cycle of repeated endogenous reinfection (endogenous autoinfection). The trophozoites remain in the apical portion of the cell (b) and undergo asexual division (merogony) to form merozoites (c). The merozoites released from type I meronts enter adjacent host cells to form type II meronts. Type II meronts do not recycle but enter host cells to form the sexual stages (d). The type II meronts form merozoites that differentiate in sexual reproductive microgamonts (male) and macrogamonts (female) (e). After fertilization, the zygote develops into resistant thick-walled oocysts (f) that are excreted from the host in the fecal material. However, some oocysts are thin-walled (g) and excyst within the same host in a process of autoinfection (h)

microgametocytes and macrogametocytes, will unite to form the zygote [3]. The diploid zygote, through a process called sporogony, will form 4 sporozoites within thick or thin-walled oocysts. The thick-walled oocyst, protected by a resistant wall, after releasing in the feces is shed into the environment, ready to infect a new individual (Figure 1) [19, 20].

Although the number of worldwide reported cases of cryptosporidiosis in the last years is increased with a number of 3 cases per 100,000 population, numerous indicators (i.e., clinical symptoms) indicate that the frequency of infection is likely to be 100 -fold higher than the number of reported cases [21]. The prevalence of Cryptosporidium infection is significantly lower in industrialized countries compared to developing countries since, in the latter, there are many people that still lack a basic level of drinking water and sanitation [22, 23]. In developing countries, cryptosporidiosis is rarely reported in immunocompetent persons, while this infection causes approximately $10-15 \%$ of cases of severe diarrheal illness mostly in malnourished children less than five years old [22, 23]. Cryptosporidium spp. have been isolated worldwide, and outbreaks of cryptosporidiosis associated with swimming pool or contaminated drinking water have been reported in several countries [24, 25]. Although there are more than 30 species of Cryptosporidium, only few species like C. hominis, C. parvum, C. meleagridis, C. felis, and C. canis are commonly found in people $[10,26,27]$. Among these species, $C$. hominis and $C$. parvum are most frequently found in intestinal infections in humans, although they differ in host range, with the former that infects exclusively humans, while the latter has an infectious cycle involving humans and ruminants, and thereby, usually C. parvum infects people who have close contact with large numbers of animals [28-30]. Although the transmission from animals is possible, it is very uncommon, and Cryptosporidium infection transmitted from sheep, horses, goats, and rodents has been rarely reported $[12,30,31]$. C. canis and $C$. felis, the dog- and the cat-adapted species, respectively, have been reported to cause infection in individuals without being sick at all $[21,30]$. However, human infections with Cryptosporidium species from pets are very rare $[10,14]$.

\section{Pathogenesis}

In immunocompetent persons, Cryptosporidium infection usually produces a bout of watery diarrhea, although the infection in some persons may not lead to the symptoms $[4,23$, 32]. The disease is likely underestimated, since the diarrhea usually resolves without any treatment [19]. Although even people who do not have direct contact with animals may be infected, those who have direct contact with infected animals (particularly calves) or swallow pool water or drink untreated water are at a higher risk of contracting cryptosporidiosis [22, 24, 25]. Cryptosporidium infections are also more common in individuals who are in poor health or who have weakened immune systems (e.g., human immunodeficiency virus (HIV)/ AIDS, cancer, and transplant patients) [19, 33-35]. Between the parasites that caused about 1 million deaths every year, cryptosporidiosis resulted in over 50,000 deaths [36, 37]. Moreover, Cryptosporidium is one of the most important protozoan pathogen that cause waterborne outbreaks worldwide [19, 32, 38]. Cryptosporidium lives in the intestines of the infected individuals and animals in the form of oocysts, which will be released in the feces [19]. After infection, the parasite 


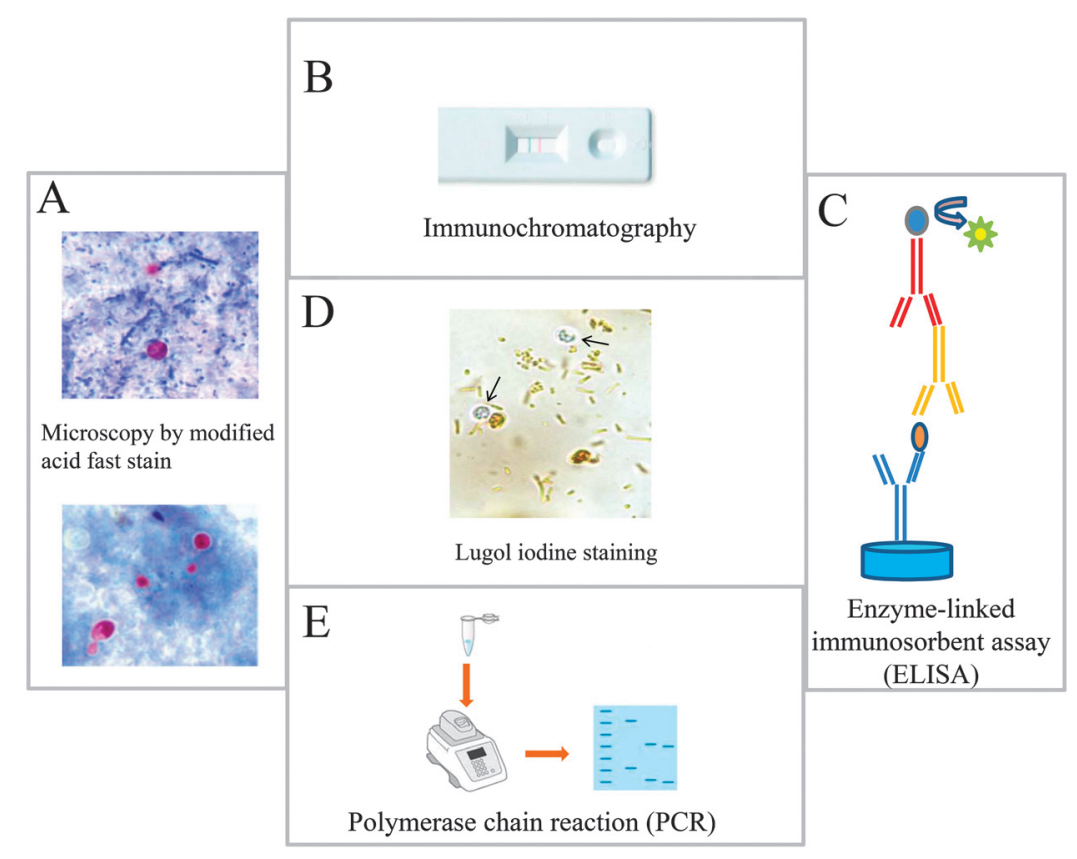

Figure 2. Principal methods used for the detection of Cryptosporidium in stool samples. (A) Microscopic identification of Cryptosporidium cysts stained by modified Ziehl-Neelsen stain. Upper panel, the oocysts stain bright red against a background of blue-green fecal debris and yeasts; lower panel, colorless oocysts that have been associated with resolving infection. (B) Immunochromatographic assay for detecting Cryptosporidium oocysts in stool samples, (C) ELISA assay for the detection of Cryptosporidium antigen in stool samples, and (D) Cryptosporidium oocysts (arrows) do not stain with Lugol's iodine solution. Oocysts appears similar to yeasts but colorless. (E) PCR detection of Cryptosporidium

alters the function of the intestinal barrier, increasing its permeability, absorption, and secretion of fluid and electrolytes, and thereby, the severity, persistence, and outcome of the infection depend on the degree of the immunocompromised status $[39,40]$. The oocysts are very resistant to chlorine, chloramines, and chlorine dioxide, which are commonly used in methods of water system disinfection, and remain vital for infection in the environment for a long time [21]. Humans become infected with Cryptosporidium by touching anything that has come in contact with contaminated feces, although the most common mode of transmission is represented by ingestion of oocysts in contaminated food and water or air [21, 40].

Recent studies indicate that cryptosporidiosis may be transmitted by inhalation of aerosolized droplets via respiratory secretions or by coughing, in addition to the well-documented fecal-oral transmission [41]. Pulmonary infections also have been reported $[41,42]$.

Immunocompromised hosts are more susceptible to infection than people with a healthy immune system, and in the subjects with HIV/AIDS, the parasite often causes a chronic, prolonged form of a disease, which is difficult to treat and can even result in death [37]. In these patients, fever and malabsorption are common, and the parasite can cause inflammatory disease of the biliary tree leading to biliary tract obstruction, sclerosing cholangitis, papillary stenosis, and pancreatitis [33, 37]. For this reason, cryptosporidiosis is considered one of the riskiest opportunistic infections for patients with acquired immune deficiency syndrome [37].

\section{Diagnosis}

The diagnosis of cryptosporidiosis is usually made by microscopic detection of the parasite oocysts, oocyst antigens, or oocyst DNA in stool samples. Since the most common symptom of cryptosporidiosis is a watery diarrhea, the differential diagnosis for Cryptosporidium includes bacterial, viral, and parasitic enteric pathogens associated with acute diarrhea such as rotaviruses, coronaviruses, Escherichia coli, and Salmonella spp. [43, 44]. However, gastrointestinal disorders may also have noninfectious causes, such as inflammatory bowel disease in humans [45].

Diagnosis of cryptosporidiosis is usually made by microscopically identifying the presence of oocysts of 4 to $6 \mu \mathrm{m}$ in diameter in the stool of the infected subjects $[44,46]$. However, since the detection of Cryptosporidium oocysts can be difficult, three fecal samples collected on separate days should be microscopically examined for detection of oocysts prior to exclude a Cryptosporidium infection in subjects with severe diarrhea [44, 45]. In addition, for detection of oocysts in stool, sample must be concentrated using the formalin-ether sedimentation method prior to microscopic examination [47]. The oocysts of Cryptosporidium can be also observed by acid-fast (modified Ziehl-Neelsen method) or phenol-auramine staining on unconcentrated fecal smears, where the oocysts stain red and bright yellow, respectively [44, 45]. However, much attention should be given to this staining since the oocysts may also appear as "ghost" cells [48]. In addition, although the oocysts of Cryptosporidium are half the size of those of Cyclospora cayetanensis (about 4-5 $\mu \mathrm{m}$ in diameter vs. 9-10 $\mu \mathrm{m}$ in diameter), another coccidian protozoan parasite that infects the intestine of humans causing acute diarrhea, much attention should be given when evaluating stool samples since the oocysts of both parasites are autofluorescent and acid-fast (Figure 2) [46, 47]. In addition, although C. cayetanensis has a life cycle similar to Cryptosporidium, its oocysts are unsporulated and not infective when shed in the feces, and thereby, direct fecal-oral transmission cannot occur [46].

Although routine diagnosis of cryptosporidiosis is generally made by the microscopic identification of oocysts in fecal smears, this method, despite being easy to use and low cost, unfortunately has a low sensitivity $(\leq 30 \%)$. Moreover, accurate diagnosis of cryptosporidiosis using this technique is largely dependent on the experience of the microscopist.

Sensitivity can be improved by performing modified acidfast stain, a staining generally performed if there are structures 
suspicious for Cryptosporidium, which has been reported to be associated with a sensitivity of $55 \%$. However, these methods cannot distinguish between Cryptosporidium species $[49,50]$. In addition to the above described methods, watery or mushy stools can be examined for the laboratory diagnosis of cryptosporidiosis using different techniques such as the enzyme-linked immunosorbent assay (ELISA) and immunochromatographic test, which have good sensitivity and specificity for detection of Cryptosporidium antigens [51-53].

Although the commercial kits have a range of sensitivities and specificities higher than that of the microscopic methods (ranging from 58 to $95 \%$ ), previous studies have shown that these antigen/antibody-based detection methods are also ineffective if the burden of this parasite in the patients is below the minimum threshold [53]. In addition, these methods are more expensive than polymerase chain reaction (PCR), which is now accepted in most laboratories as the gold standard for the detection of this parasite in the stool. Previous studies have shown that compared to PCR, microscopy, ELISA, and immunochromatographic test (ICT) are less convenient in terms of cost, sensitivity, and specificity and also are more time consuming [54-56]. Although there have been important advances in diagnostic tools (i.e., the availability of multiplex PCR assays for the detection of intestinal protozoa), the accessibility to this molecular technique is limited in some labs and totally absent in others. In addition, the expense and requirement for technical expertise have limited their use particularly in high-prevalence regions such as developing countries.

\section{Conclusion}

Despite the global prevalence and the high impact of cryptosporidiosis, principally in immunocompromised patients, major deficiencies exist in the current control programs, especially in terms of available diagnostic tools. In addition, most common diagnostic tests tend to misdiagnose the disease in endemic areas. In particular, microscopic techniques are the most widely used method for detection of cryptosporidia in stool samples, and the diagnostic accuracy of these methods is largely dependent on the experience of the microscopist.

Since early diagnosis is the best way to fight the infection, there is a need to develop molecular techniques that are sensitive, specific, easy to perform, cost-effective, and highthroughput.

\section{Funding Sources}

This work was supported by Fondo per il Finanziamento delle Attività Base di Ricerca (FFBRA2017)

\section{Authors' Contributions}

All authors conceived the original idea, CB wrote the manuscript with support from EG and VML.

\section{Conflict of Interest}

The authors declare no conflict of interest.

\section{References}

1. Dillingham RA, Lima AA, Guerrant RL. Cryptosporidiosis: epidemiology and impact. Microbes Infect. 2002;4(10):1059-66.

2. Tzipori S. Introduction. Cryptosporidiosis: current trends and challenges. Microbes Infect. 2002;4(10):1045.

3. Tzipori S, Ward H. Cryptosporidiosis: biology, pathogenesis and disease. Microbes Infect. 2002;4(10):1047-58

4. Khalil IA, Troeger C, Rao PC, Blacker BF, Brown A, Brewer TG, et al. Morbidity, mortality, and long-term consequences associated with diarrhoea from Cryptosporidium infection in children younger than 5 years: a metaanalyses study. Lancet Glob Health. 2018;6(7):e758-68.
5. Tzipori S, Widmer G. A hundred-year retrospective on cryptosporidiosis. Trends Parasitol. 2008;24(4):184-9.

6. Meisel JL, Perera DR, Meligro C, Rubin CE. Overwhelming watery diarrhea associated with a cryptosporidium in an immunosuppressed patient. Gastroenterology. 1976;70(6):1156-60

7. Nime FA, Burek JD, Page DL, Holscher MA, Yardley JH. Acute enterocolitis in a human being infected with the protozoan Cryptosporidium. Gastroenterology. 1976;70(4):592-8.

8. Fayer R, Ungar BL. Cryptosporidium spp. and cryptosporidiosis. Microbiol Rev. 1986;50(4):458-83.

9. Majeed QAH, El-Azazy OME, Abdou NMI, Al-Aal ZA, El-Kabbany AI, Tahrani LMA, et al. Epidemiological observations on cryptosporidiosis and molecular characterization of Cryptosporidium spp. in sheep and goats in Kuwait. Parasitol Res. 2018;117(5):1631-6.

10. Ryan U, Fayer R, Xiao L. Cryptosporidium species in humans and animals: current understanding and research needs. Parasitology. 2014;141(13):1667-85.

11. Brankston G, Boughen C, Ng V, Fisman DN, Sargeant JM, Greer AL. Assessing the impact of environmental exposures and Cryptosporidium infection in cattle on human incidence of cryptosporidiosis in Southwestern Ontario, Canada. PLoS One. 2018;13(4):e0196573.

12. Thomson S, Hamilton CA, Hope JC, Katzer F, Mabbott NA, Morrison $\mathrm{LJ}$, et al Bovine cryptosporidiosis: impact, host-parasite interaction and control strategies. Vet Res. 2017;48(1):42.

13. Zahedi A, Paparini A, Jian F, Robertson I, Ryan U. Public health significance of zoonotic Cryptosporidium species in wildlife: Critical insights into better drinking water management. Int $\mathrm{J}$ Parasitol Parasites Wildl. 2016;5(1):88-109.

14. Leitch GJ, He Q. Cryptosporidiosis-an overview. J Biomed Res. 2012;25(1):1-16.

15. Suarez CE, Bishop RP, Alzan HF, Poole WA, Cooke BM. Advances in the application of genetic manipulation methods to apicomplexan parasites. Int J Parasitol. 2017;47(12):701-10

16. Karanis P. The truth about in vitro culture of Cryptosporidium species. Parasitology. 2018;145(7):855-64.

17. Pumipuntu N, Piratae S. Cryptosporidiosis: A zoonotic disease concern. Vet World. 2018;11(5):681-6.

18. Chalmers RM, Smith R, Elwin K, Clifton-Hadley FA, Giles M: Epidemiology of anthroponotic and zoonotic human cryptosporidiosis in England and Wales, 2004-2006. Epidemiol Infect. 2011;139(5):700-12.

19. Bouzid M, Hunter PR, Chalmers RM, Tyler KM. Cryptosporidium pathogenicity and virulence. Clin Microbiol Rev. 2013;26(1):115-34.

20. Jenkins MB, Eaglesham BS, Anthony LC, Kachlany SC, Bowman DD, Ghiorse WC. Significance of wall structure, macromolecular composition, and surface polymers to the survival and transport of Cryptosporidium parvum oocysts. Appl Environ Microbiol. 2010;76(6):1926-34.

21. Shrivastava AK, Kumar S, Smith WA, Sahu PS. Revisiting the global problem of cryptosporidiosis and recommendations. Trop Parasitol. 2017;7(1):8-17.

22. Bouzid M, Kintz E, Hunter PR. Risk factors for Cryptosporidium infection in low and middle income countries: A systematic review and metaanalysis. PLoS Neglected Trop Dis. 2018;12(6):e0006553.

23. Shoultz DA, de Hostos EL, Choy RK. Addressing Cryptosporidium Infection among Young Children in Low-Income Settings: The Crucial Role of New and Existing Drugs for Reducing Morbidity and Mortality. PLoS Neglected Trop Dis. 2016;10(1):e0004242.

24. Fayer R, Farley CA, Lewis EJ, Trout JM, Graczyk TK. Potential Role of the Eastern Oyster, Crassostrea virginica, in the Epidemiology of Cryptosporidium parvum. Appl Environ Microbiol. 1997;63(5):2086-8.

25. Fayer R, Morgan U, Upton SJ. Epidemiology of Cryptosporidium: transmission, detection and identification. Int J Parasitol. 2000;30(1213):1305-22.

26. Ayinmode AB, Oliveira BCM, Obebe OO, Dada-Adgebola HO, Ayede AI, Widmer G. Genotypic Characterization of Cryptosporidium Species in Humans and Peri-Domestic Animals in Ekiti and Oyo States, Nigeria. J Parasitol. 2018;104(6):639-44.

27. Slapeta J: Cryptosporidiosis and Cryptosporidium species in animals and humans: a thirty colour rainbow? Int J Parasitol. 2013;43(12-13):95770 .

28. Dixon B, Parrington L, Cook A, Pintar K, Pollari F, Kelton D, et al. The potential for zoonotic transmission of Giardia duodenalis and Cryptosporidium spp. from beef and dairy cattle in Ontario, Canada. Vet Parasitol. 2011;175(1-2):20-6.

29. Ehsan AM, Geurden T, Casaert S, Parvin SM, Islam TM, Ahmed UM, et al. Assessment of zoonotic transmission of Giardia and Cryptosporidium between cattle and humans in rural villages in Bangladesh. PLoS One. 2015;10(2):e0118239.

30. Hunter PR, Thompson RC. The zoonotic transmission of Giardia and Cryptosporidium. Int J Parasitol. 2005;35(11-12):1181-90.

31. Chalmers RM, Davies AP: Minireview: clinical cryptosporidiosis. Exp Parasitol. 2010;124(1):138-46.

32. Adler S, Widerstrom M, Lindh J, Lilja M. Symptoms and risk factors of Cryptosporidium hominis infection in children: data from a large waterborne outbreak in Sweden. Parasitol Res. 2017;116(10):2613-18.

33. Wang ZD, Liu Q, Liu HH, Li S, Zhang L, Zhao YK, et al. Prevalence of Cryptosporidium, microsporidia and Isospora infection in HIV-infected people: a global systematic review and meta-analysis. Parasites Vectors. 2018;11(1):28

34. Samie A, Makuwa S, Mtshali S, Potgieter N, Thekisoe O, Mbati P, et al. Parasitic infection among HIV/AIDS patients at Bela-Bela clinic, Limpopo province, South Africa with special reference to Cryptosporidium. Southeast Asian J Trop Med Public Health. 2014;45(4):783-95. 
35. Florescu DF, Sandkovsky U. Cryptosporidium infection in solid organ transplantation. World J Transplant. 2016;6(3):460-71.

36. Shirley DA, Moonah SN, Kotloff KL. Burden of disease from cryptosporidiosis. Curr Opin Infect Dis, 2012;25(5):555-63.

37. Wang RJ, Li JQ, Chen YC, Zhang LX, Xiao LH. Widespread occurrence of Cryptosporidium infections in patients with HIV/AIDS Epidemiology, clinical feature, diagnosis, and therapy. Acta Trop 2018, 187:257-63.

38. Rehn M, Wallensten A, Widerstrom M, Lilja M, Grunewald M, Stenmark S, et al. Post-infection symptoms following two large waterborne outbreaks of Cryptosporidium hominis in Northern Sweden, 2010-2011. BMC Public Health. 2015;15:529.

39. Kumar A, Chatterjee I, Anbazhagan AN, Jayawardena D, Priyamvada $\mathrm{S}$, Alrefai WA, et al. Cryptosporidium parvum disrupts intestinal epithelial barrier function via altering expression of key tight junction and adherens junction proteins. Cell Microbiol. 2018;20(6):e12830.

40. Petry F, Jakobi V, Tessema TS Host immune response to Cryptosporidium parvum infection. Exp Parasitol. 2010;126(3):304-9.

41. Sponseller JK, Griffiths JK, Tzipori S. The evolution of respiratory Cryptosporidiosis: evidence for transmission by inhalation. Clin Microbio Rev. 2014;27(3):575-86.

42. Reina FT, Ribeiro CA, Araujo RS, Matte MH, Castanho RE, Tanaka II, et al. Intestinal and Pulmonary Infection by Cryptosporidium Parvum in Two Patients with HIV/AIDS. Rev Inst Med Trop Sao Paulo. 2016;58:21.

43. Mehta P. Laboratory diagnosis of cryptosporidiosis. J Postgrad Med. 2002;48(3):217.

44. Khurana S, Chaudhary P. Laboratory diagnosis of cryptosporidiosis. Trop Parasitol. 2018;8(1):2-7.

45. Omoruyi BE, Nwodo UU, Udem CS, Okonkwo FO. Comparative diagnostic techniques for cryptosporidium infection. Molecules. 2014;19(2):2674-83.

46. Ahmed SA, Karanis P. Comparison of current methods used to detect Cryptosporidium oocysts in stools. Int J Hyg Environ Health. 2018;221(5):743-63. 47. Pacheco FT, Silva RK, Martins AS, Oliveira RR, Alcantara-Neves NM, Silva MP, et al. Differences in the detection of Cryptosporidium and Isospora (Cystoisospora) oocysts according to the fecal concentration or staining method used in a clinical laboratory. J Parasitol. 2013;99(6):1002-8.
48. Vanathy K, Parija SC, Mandal J, Hamide A, Krishnamurthy S. Detection of Cryptosporidium in stool samples of immunocompromised patients. Trop Parasitol. 2017;7(1):41-6.

49. Elsafi SH, Al-Maqati TN, Hussein MI, Adam AA, Hassan MM, Al Zahrani EM. Comparison of microscopy, rapid immunoassay, and molecular techniques for the detection of Giardia lamblia and Cryptosporidium parvum. Parasitol Res. 2013;112(4):1641-6.

50. McHardy IH, Wu M, Shimizu-Cohen R, Couturier MR, Humphries RM. Detection of intestinal protozoa in the clinical laboratory. J Clin Microbiol. 2014;52(3):712-20.

51. Agnamey P, Sarfati C, Pinel C, Rabodoniriina M, Kapel N, Dutoit E, et al. Evaluation of four commercial rapid immunochromatographic assays for detection of Cryptosporidium antigens in stool samples: a blind multicenter trial. J Clin Microbiol. 2011;49(4):1605-7.

52. Christy NC, Hencke JD, Escueta-De Cadiz A, Nazib F, von Thien H, Yagita K, et al. Multisite performance evaluation of an enzyme-linked immunosorbent assay for detection of Giardia, Cryptosporidium, and Entamoeba histolytica antigens in human stool. J Clin Microbiol. 2012;50(5):1762-3.

53. Hawash Y. Evaluation of an immunoassay-based algorithm for screening and identification of giardia and cryptosporidium antigens in human faecal specimens from saudi arabia. J Parasitol Res. 2014;2014:213745.

54. Friesen J, Fuhrmann J, Kietzmann H, Tannich E, Muller M, Ignatius R. Evaluation of the Roche LightMix Gastro parasites multiplex PCR assay detecting Giardia duodenalis, Entamoeba histolytica, cryptosporidia, Dientamoeba fragilis, and Blastocystis hominis. Clin Microbiol Infect. 2018;24(12):1333-7.

55. Laude A, Valot S, Desoubeaux G, Argy N, Nourrisson C, Pomares C, et al. Is real-time PCR-based diagnosis similar in performance to routine parasitological examination for the identification of Giardia intestinalis, Cryptosporidium parvum/Cryptosporidium hominis and Entamoeba histolytica from stool samples? Evaluation of a new commercial multiplex PCR assay and literature review. Clin Microbiol Infect. 2016;22(2):190 e191-8.

56. Autier B, Belaz S, Razakandrainibe R, Gangneux JP, Robert-Gangneux F. Comparison of three commercial multiplex PCR assays for the diagnosis of intestinal protozoa. Parasite. 2018;25:48. 